\title{
Transplante autólogo de células-tronco hematopoiéticas sem uso de hemocomponentes
}

\author{
Hematopoietic stem cell transplantation without the use of blood transfusions
}

Roberto L. Silva

Maria Cristina M. A. Macedo

\begin{abstract}
O transplante de células-tronco hematopoéticas (TCTH) é terapia consolidada para tratamento de algumas doenças onco-hematológicas, e o suporte transfusional tem, tradicionalmente, sido fundamental para a realização do mesmo. Descrevemos um caso de paciente testemunha de Jeová, portadora de linfoma Hodgkin em terceira remissão parcial, que foi submetida a quimioterapia de altas doses com regime de condicionamento clássico (carmustina, etoposide, citarabina, melfalan) e posterior infusão de células-tronco hematopoéticas sem o uso de hemocomponentes. A paciente apresentou toxicidade hematológica inerente ao procedimento e medidas clínicas de suporte tais como a utilização de eritropoetina, IL 11, antifibrinolítico, entre outras, foram utilizadas na tentativa de minimizar o risco de sangramento e anemia grave. $O$ curso do transplante transcorreu sem complicações graves. Este caso demonstra que o transplante autólogo de células-tronco hematopoéticas sem o uso de hemocomponentes é factível em situações especiais, onde há clara expressão do desejo do paciente associado a condições clínicas favoráveis e acompanhamento médico especialista rigoroso. Rev. bras. hematol. hemoter. 2006;28(2):153-156.
\end{abstract}

Palavras-chave: Transplante de células-tronco hematopoéticas;linfoma Hodgkin.

\section{Introdução}

O transplante de células-tronco hematopoéticas (TCTH) é uma terapia consagrada no tratamento de doenças oncohematológicas e tem se mostrado o tratamento de maior eficácia na recaída de algumas delas como o linfoma Hodgkin e não-Hodgkin. ${ }^{1}$ A quimioterapia de altas doses é o princípio deste tratamento e a finalidade é superar a resistência das células neoplásicas. A mieloablação com pancitopenia grave é o efeito colateral óbvio e esta tem sido controlada com suporte clínico e transfusional. O manejo transfusional neste procedimento tem sido baseado principalmente na profilaxia de sangramentos e dos sintomas de anemia.

O uso de fatores de crescimento de leucócitos e plaquetas e eritropoetina, assim como a administração de antifibrinolíticos e pró-coagulantes, tem propiciado a redução no uso de hemocomponentes em pacientes oncológicos e hematológicos, ${ }^{2,3}$ já que estes também não são isentos de complicações. ${ }^{4}$

Por motivações religiosas, cerca de 1.600 .000 pessoas no Brasil ${ }^{5,6}$ recusam-se a receber transfusão sangüínea e ficam, portanto, excluídas da possibilidade de receber diversas terapias, principalmente procedimentos cirúrgicos. Ballen et al. ${ }^{7}$ e Mazza et al. ${ }^{8}$ relataram casos de transplante de medula óssea realizados sem o uso de hemocomponentes com toxicidade relacionada ao tratamento semelhante aos descritos na literatura.

Neste trabalho relatamos um caso em que foi utilizada quimioterapia de altas doses com resgate de células-tronco hematopoéticas periféricas, com sucesso, em uma menor portadora de linfoma de Hodgkin sem o uso de hemocomponentes.

Divisão de Transplante de Células-Tronco Hematopoéticas do Hospital e Maternidade São Camilo da Pompéia.

Divisão de Transplante de Medula Óssea da equipe Bio Sana`s.

Correspondência: Roberto Luiz da Silva.

Rua Vergueiro 2949, conjunto 71 - Vila Mariana

04101-300 - São Paulo-SP - Brasil

Tel. (011) 55746794

E-mail: biosanas@uol.com.br 


\section{Relato do caso}

P.A.B., 14 anos, teve o diagnóstico de histiocitose realizado em dezembro de 1997 e foi tratada por equipe de oncopediatria com diversos protocolos até junho/2001 sem resposta adequada. Em julho/01 foi realizada revisão do diagnóstico que mostrou tratar-se de linfoma de Hodgkin esclerose nodular e a paciente iniciou tratamento com OPPA/ ABVD (vincristina, prednisona, procarbazina e adriamicina / doxorrubicina, bleomicina, vimblastina e dacarbazina) até novembro/2001, tendo obtido finalmente primeira remissão. Apresentou recaída da doença 2 anos e 7 meses após este tratamento, sendo realizado resgate com um ciclo de ifosfamida e vepesid em setembro/04 sem resposta, sendo substituído resgate para COMP (ciclofosfamida, vincristina, metotrexate e prednisona) de outubro/04 a janeiro de 2005 com obtenção de segunda remissão, porém apresentou nova progressão da doença em março/05. Optou-se pela realização de resgate com cisplatina, gemcitabina e navelbine, três ciclos, com obtenção de terceira remissão parcial. A paciente foi então encaminhada para tratamento com TCTH, já que esta foi considerada a melhor alternativa para o controle e/ou cura da doença.

\section{Método de mobilização}

A coleta de células-tronco hematopoéticas periféricas foi obtida em duas etapas, entre a segunda e terceira últimas quimioterapias de resgate (agosto/ 2005) e após esta última (setembro/2005). A mobilização das células-tronco ocorreu após estímulo com GCSF na dose de $20 \mathrm{mcg} / \mathrm{kg} / \mathrm{dia}$ (divididos em duas doses) por cinco dias, segundo descrição de Sullivan. ${ }^{9} \mathrm{O}$ número total de células CD34 obtido foi 6.1 x $10^{6}$ /céls CD34/kg de peso $\left(2,27 \times 10^{6}\right.$ na primeira coleta e $3,83 \times 10^{6}$ na segunda coleta).

Transplante de células-tronco periféricas

A paciente foi submetida ao TCTH após a obtenção de consentimento esclarecido com a paciente e pais. O regime de condicionamento utilizado foi BEAM (carmustina $300 \mathrm{mg} /$ $\mathrm{m}^{2}$, etoposide $400 \mathrm{mg} / \mathrm{m}^{2}$, cytarabina $800 \mathrm{mg} / \mathrm{m}^{2}$ e melfalano $140 \mathrm{mg} / \mathrm{m}^{2}$ ). As células-tronco hematopoéticas criopreservadas foram infundidas em dois dias consecutivos para que não houvesse infusão de grande quantidade do criopreservante (dimetilsulfóxido).

\section{Suporte clínico e hematológico}

O TCTH foi iniciado após obtenção de níveis de hemoglobina de 12 g/dl. Foi utilizada IL 11 (50 mcg/kg/dia) do +1 ao +13 do TMO, eritropoetina (40.000 unidades uma vez por semana) e GCSF ( $5 \mathrm{mcg} / \mathrm{kg} / \mathrm{dia}$ ) do +11 ao +16 do TMO. As citocinas utilizadas foram baseadas em protocolo previamente descrito por Ballen ${ }^{7}$ e Mazza. ${ }^{8}$

Foi realizada reposição de ferro, ácido folínico e vitami- na B12. Durante o período de mucosite, que se deu a partir do +3, iniciamos reposição de vitamina K endovenosa. Para diminuir a espoliação sanguínea, evitamos a realização de exames sistematicamente e os coletamos nos dias $-2,+4,+7,+10$, $+11,+14,+16$ e +19 . Os resultados obtidos podem ser vistos na tabela abaixo. No +11 do TCTH, após quadro febril, a paciente evoluiu com petéquias de membros inferiores e discreto sangramento gengival sendo introduzido neste momento ácido épsilon- aminocapróico $200 \mathrm{mg} / \mathrm{kg} /$ dia dividido em 4 doses, além do uso tópico. Houve controle imediato do sangramento gengival e, após 48 horas, desaparecimento das petéquias.

A paciente evoluiu com sintomas de anemia, apresentando aumento da freqüência cardíaca, mas sem descompensação hemodinâmica. Os leucócitos iniciaram recuperação a partir do +10, porém somente tivemos documentação de enxertia no dia +14 do TCTH. Teve alta hospitalar no dia + 19 do TCTH.

Tabela

\begin{tabular}{ccccccccccc}
\hline$*$ & Pré-TCTH & -2 & +4 & +7 & +10 & +11 & +14 & +16 & +19 & +35 \\
\hline $\begin{array}{c}\text { Hemoglobina } \\
(\mathrm{g} / \mathrm{dl})\end{array}$ & 12 & 10,9 & 8,4 & 7,1 & 6,8 & 7,1 & 8,2 & 6,7 & 8,7 & 11,3 \\
$\begin{array}{c}\text { Leucócitos } \\
\left(\mathrm{x} 10^{3} / \mu \mathrm{l}\right)\end{array}$ & 9.5 & 3.1 & 0.4 & 0.3 & 0.8 & 1.1 & 2.1 & 7.0 & 4.5 & 5.9 \\
$\begin{array}{c}\text { Granulócitos } \\
\left(\mathrm{x} 10^{3} / \mu \mathrm{l}\right)\end{array}$ & 7.4 & 2.5 & 0 & 0 & 0 & 0.3 & 0.9 & 4.4 & 2.9 & 2.0 \\
$\begin{array}{c}\text { Plaquetas } \\
\left(\mathrm{x} 10^{3} / \mu \mathrm{l}\right)\end{array}$ & 214 & 212 & 60 & 33 & 58 & 28 & 49 & 68 & 91 & 72 \\
\hline
\end{tabular}

\section{Discussão}

Não pretendemos neste relato discutir os aspectos religiosos envolvidos na questão. No entanto, é de conhecimento da comunidade médica que cerca de 6 milhões de pessoas no mundo são adeptas do grupo religioso Testemunhas de Jeová, ${ }^{5,6}$ que não aceitam transfusão de sangue total ou hemocomponentes (glóbulos vermelhos, glóbulos brancos, plaquetas ou plasma), sendo que o uso de alguns hemoderivados (ou frações sangüíneas como albumina, imunoglobulinas e fatores de coagulação) podem ser aceitos por alguns destes pacientes. ${ }^{10}$ Este aspecto certamente tem excluído muitos destes cidadãos de um tratamento adequado em diversos centros médicos no Brasil. Durante o transplante de medula óssea autólogo, normalmente são utilizadas cinco a vinte transfusões de concentrados de glóbulos ou plaquetas. ${ }^{11}$ Alguns centros vêm realizando protocolos especiais na tentativa de minimizar e até mesmo deixar de utilizar hemocomponentes, ${ }^{7,8,12,13,14}$ entretanto, não foi documentado nenhum caso de sucesso entre menores de 18 anos.

A quimioterapia de altas doses com resgate de célulastronco foi um grande avanço no controle de doenças neoplásicas e tem se mostrado o principal recurso no controle de recaídas de linfomas Hodgkin e não-Hodgkin. O fundamento do tratamento, entretanto, baseia-se na possibilida- 
de de extrapolar os limites da toxicidade hematológica, suportando os efeitos desta, por período limitado, com o uso dos hemocomponentes, entre outros. Tradicionalmente, transfusões são prescritas a partir de valores preestabelecidos para se evitarem sintomas de anemia e principalmente riscos de sangramentos. ${ }^{15}$ A maior parte dos centros utiliza o valor de 20.000 plaquetas por $\mathrm{mm}^{3}$ para indicar transfusão, ${ }^{11}$ no entanto, Gmur et al. ${ }^{16}$ determinaram que o limite para transfusões profiláticas de plaquetas em pacientes com leucemia aguda poderia ser de $5.000 / \mathrm{mm}^{3}$ para pacientes não complicados, $10.000 / \mathrm{mm}^{3}$ para pacientes com febre ou sangramentos e $20.000 / \mathrm{mm}^{3}$ para pacientes com coagulopatia. O valor estipulado para as transfusões, entretanto, é variável de acordo com a experiência dos diversos serviços e o uso racional de hemoderivados, baseados no contexto clínico, tem sido defendido por alguns autores. ${ }^{17} \mathrm{O}$ uso de interleucina 11 reduziu o consumo de plaquetas em quimioterapia convencional, reduzindo o tempo e a gravidade da plaquetopenia, embora estes dados não sejam conclusivos no contexto do TCTH. ${ }^{3,18} \mathrm{O}$ uso da eritropoetina, da mesma forma, parece diminuir a necessidade do uso de hemáceas, e trabalhos em quimioterapia convencional tem mostrado redução de até $50 \%$ das necessidades transfusionais com o uso da mesma. ${ }^{2}$ Já no contexto do TCTH, parece haver maior benefício do uso da eritropoetina no TCTH alogênico. ${ }^{19,20}$ Optamos por limitar o uso do GCSF já que este pode estar associado a menor recuperação plaquetária como sugerido em um estudo em transplantes. ${ }^{11} \mathrm{~A}$ reposição de ferro associada ao uso da eritropoetina foi realizada seguindo protocolo já descrito ${ }^{7}$ já que tem sido importante na manutenção dos estoques de ferro e recuperação medular. ${ }^{21}$ A retirada diária de sangue para realização de exames pode levar à espoliação de cerca de $40 \mathrm{ml}$ de sangue por dia em pacientes críticos, o que certamente contribui para um aumento da necessidade transfusional nestes pacientes. ${ }^{22}$ Neste caso, procuramos reduzir as coletas sangüíneas, trabalhando com o menor volume necessário para realização de exames que, por sua vez, também foram limitados, o que certamente reduziu o consumo, como já descrito por Ford. ${ }^{23} \mathrm{O}$ ácido aminocapróico é um agente antifibrinolítico que, utilizado em cirurgias, levou à diminuição da perda sangüínea ${ }^{24}$ e que demonstrou diminuir o sangramento mucoso em pacientes plaquetopênicos. ${ }^{25}$ Neste caso foi usado com sucesso no momento de sangramento gengival.

A opção pela realização deste TCTH, apesar da restrição ao uso de hemocomponentes, levou em consideração a indicação precisa, a idade da paciente, a ausência de comorbidades associadas e o número adequado de células-tronco periféricas coletadas.

Este é, segundo o nosso conhecimento, o primeiro caso relatado de TCTH sem o uso de transfusão sangüínea realizado com sucesso em criança e o primeiro relato no Brasil. A objeção pessoal à transfusão sangüínea, antes de representar um obstáculo ao tratamento médico adequado, pode representar um desafio para o desenvolvimento de alternativas terapêuticas, contribuindo assim para o avanço da medicina.

\section{Conclusão}

O transplante autólogo de células-tronco hematopoéticas sem o uso de hemocomponentes é factível em situações especiais, onde há clara expressão do desejo do paciente associado a condições clínicas favoráveis e acompanhamento médico especialista rigoroso.

\section{Abstract}

Hematopoietic stem cell transplantation (HSCT) is standard therapy for the treatment of some hematological neoplasms and support with blood transfusions is considered essential for this procedure. Herein we describe the case of a Jeovah's witness who had Hodgkin's lymphoma in third partial remission and was submitted to high-dose chemotherapy using a classic conditioning regimen (carmustine, etoposide, cytarabine, melphalan) with posterior infusion of autologous peripheral blood stem cells without the use of blood transfusions. The patient had the usual degree of hematological toxicity and was treated with clinical support measures, such as the use of erythropoietin, IL-11 and antifibrinolytics, with the goal of minimizing the risk of bleeding and serious anemia. The HSTC coursed without major complications. This report shows that the autologous peripheral blood stem cell transplantation without the use of blood and platelet transfusions is feasible in special situations, where patients clearly express their wishes and where there is a favorable clinical condition with rigorous medical care provided by specialists. Rev. bras. hematol. hemoter. 2006;28(2):153-156.

Key words: Hematopoietic stem cell transplantation; Hodgkin's lymphoma.

\section{Agradecimentos}

Prof. Dr. Wilson Modesto Pollara - Diretor clínico do Hospital e Maternidade São Camilo Pompéia.

Dr. Fábio Pires de Souza Santos - Médico da Divisão de Onco-hematologia e Transplante de Medula Óssea da Bio Sana's. Sr. Guilherme Rabello - Divisão de Pesquisa da Comissão de Ligação Hospitalar para Testemunhas de Jeová (COLIH).

Sr. Pedro Roberto Gomes Rodrigues - Assessor comercial do Hospital e Maternidade São Camilo Pompéia.

Equipe de Enfermagem do Departamento de Transplante de Medula Óssea do Hospital e Maternidade São Camilo Pompéia. Equipe Multidisciplinar da Bio Sana's Serviços Médicos S/S. Dra. Maria Lydia Mello de Andréa - Diretora do Serviço de Oncologia do Hospital Darcy Vargas.

\section{Referências Bibliográficas}

1. Lancet JE, Rapoport AP, Brasacchio R, et al. Autotransplantation for relapsed or refractory Hodgkin's disease: long term follow-up and analysis of prognostic factors. Bone Marrow Transplantation 1998;22:265-71.

2. Glossmann JP, Engert A, Wassmer G, et al. Recombinant human erythropoietin, epoetin beta, in patients with relapsed lymphoma treated with aggressive sequential salvage chemotherapy-results of a randomized trial. Ann Hematol 2003;82:469-75. 
3. Tepler I, Elias L, Smith JW, et al. A randomized placebo-controlled trial of recombinant human IL11 in cancer patients with severe thrombocytopenia due to chemotherapy. Blood 1996;87:3.607-14.

4. Sandler SG, Yu H, Rassai N. Risk of blood transfusion and their prevention. Clin Adv Hematol Oncol 2003;1:307-13.

5. Anuário de 2005 das Testemunhas de Jeová, páginas 31-33

6. A Sentinela de 1\%/2/05, páginas 19-22, publicados pela Associação Torre de Vigia de Bíblias e Tratados.

7. Ballen KK, Becker PS, Yeap BY, et al. Autologous stem cell transplantation can be performed safely without the use of bloodproduct support. J Clin Oncol 2004;22:4087-94.

8. Mazza P, Prudenzano A, Amurre B, et al. Myeloablative therapy and bone marrow transplantation in Jehovah's Witnesses with malignancies: single center experience. Bone Marrow Transplantation 2003;00:1-4.

9. Sullivan M, Mathews B, Ford P. Stem cell transplantation without the use of blood products. Updates in Blood Conservation and Transfusion Alternatives 2003;1:5-11. Official publication of the Australasian Association for Blood Conservation (AABC).

10. A Sentinela de 1\%6/04, páginas 19-24 e 29-31, publicados pela Associação Torre de Vigia de Bíblias e Tratados

11. Bernstein SH, Nademanee AP, Vose JM, et al. A multicenter study of platelet recovery and utilization in patients after myeloablative therapy and hematopoetic stem cell transplantation. Blood 1998; 91:3509-17.

12. Zenz T, Döhner H, Bunjes D. Transfusion-free reduced intensity conditioned allogeneic stem cell transplantation in a Jehovah's Witnesses. Bone Marrow Transplantation 2003;00:1-2.

13. Ruiz-Arguelles GJ, Morales-Toquero A, Lopez-Martinez B, et al. Bloodless (transfusion free) hematopoietic stem cell transplants: the Mexican experience. Bone Marrow Transplantation 2005; 36:715-20.

14. Wandt H, Schaefer-Eckartk, Wilhelm M. Two allogeneic hematopoietic stem cell transplantations without the use of blood-product support. Haematologica 2005;90:1.292-4.

15. Gaydos LA, Freirech EJ, Mantel N. The quantitative relation between platelet count and hemorrhage in patients with acute leukemia. N England J Med 1962;266:905-9.

16. Gmur J, Burger J, Schanzu, et al. Safety of stringent prophylactic platelet transfusion policy for patients with acute leukaemia. Lancet 1991;338:1.223-6.

17. Wandt H, Ehninger G, Gallmeier WM. New strategies for prophylactic platelet transfusion in patients with hematologic diseases. The Oncologist 2001;6:446-50

18. Vredenburgh JJ, Hussein A, Fisher D, et al. A randomised trial of recombinant human IL11 following autologous bone marrow transplantation with peripheral blood progenitor cell support in patients with breast cancer. Biology Transplant 1998;4:134-41.

19. Klaesson S, Ringden O, Ljungman P, et al. Reduced blood transfusion requirements after allogeneic bone marrow transplantation: Results of a randomized, double blind study with high dose erythropoietin. Bone Marrow Transplant 1994;13:397-402.

20. Ayash LJ, Elias A, Hunt M, et al. Recombinant erythropoietin for the treatment of the anemia associated with autologous bone marrow transplantation. Br J Haematol 1994;87:153-161.

21. Gooldnough LT, Skikne B, Brugnara C, et al. Erythropoietin, iron and erythropoiesis. Blood 2000;96:823-33.

22. Smoller BR, Kruskall MS. Phlebotomy for diagnostic laboratory tests in adults. N Engl J Med 1986;314:1.233-35.

23. Ford PA, Borghaei J, Henry DH. Treatment of patients who refuse blood components, in Alving BM (ed): Blood components and pharmacologic agents in the treatment of congenital and acquired bleeding disorders. Bethesda MD AABB Press 2000.
24. Vander STJ, Kaur S, Lancey RA, et al. Reduction of bleeding after heart operations through the prophylactic use of epsilonaminocaproic acid. J Thorac Cardiovasc Surg 1996;112:1098-1107.

25. Bartholomew JR, Salgia R, Bell WR. Control of bleeding in patients with immune and non-immune thrombocytopenia with aminocaproic acid. Arch Intern Med 1989;149:1959-61.

Avaliação: Editor e dois revisores externos.

Conflito de interesse: não declarado

Recebido: 23/12/2005

Aceito após modificações: 09/03/2006 